\title{
Amphotericin B lipid formulations and target-site penetration into human body fluids
}

Stefan Weiler ${ }^{1}$, Rosa Bellmann-Weiler ${ }^{2}$, Stefan Dunzendorfer ${ }^{3}$, Michael Joannidis ${ }^{3}$ and Romuald Bellmann*1,3

\author{
Address: ${ }^{1}$ Clinical Pharmacokinetics Unit, Laboratory of Inflammation Research, Department of Internal Medicine I, Innsbruck Medical \\ University, 6020 Innsbruck, Austria, 2Infectious Diseases, Department of Internal Medicine I, Innsbruck Medical University, 6020 Innsbruck, \\ Austria and ${ }^{3}$ Intensive Care Unit, Department of Internal Medicine I, Innsbruck Medical University, 6020 Innsbruck, Austria \\ Email: Romuald Bellmann* - romuald.bellmann@i-med.ac.at \\ * Corresponding author
}

from I4th Scientific Symposium of the Austrian Pharmacological Society (APHAR)

Innsbruck, Austria. 21-22 November 2008

Published: 5 November 2008

BMC Pharmacology 2008, 8(Suppl I):A58 doi:I0.II86/I47I-22I0-8-SI-A58

This abstract is available from: http://www.biomedcentral.com/I47I-22I0/8/SI/A58

(c) 2008 Weiler et al; licensee BioMed Central Ltd.

\section{Background}

Amphotericin B (AmB) lipid formulations have been introduced in antifungal therapy to reduce the toxicity of AmB. The lipid-associated fraction of liposomal AmB (LAMB), AmB colloidal dispersion (ABCD) and AmB lipid complex (ABLC) exhibit different compositions, structures, and particle sizes, which are reflected in different plasma pharmacokinetics. Antimycotic drug concentrations at target-site can be essential for clinical response.

\section{Materials and methods}

Concentrations of the three therapeutically used lipid formulations were determined in human body fluids and compared to previous data from tissue samples and epithelial lining fluid. AmB levels in pleural effusion of seven patients, ascites of three patients and bile fluid obtained from one patient were analysed after administration of a lipid formulation. In order to calculate the penetration ratio the concentrations in pleural effusion and bile fluid were compared with simultaneous levels of lipid formulated AmB in plasma. All samples were purified by solid phase extraction and AmB was quantified by HPLC.

\section{Results}

Total AmB levels in pleural effusion ranged from $0.02 \mathrm{~g} / \mathrm{L}$ to $0.43 \mathrm{~g} / \mathrm{L}$. Mean levels of $0.22 \pm 0.09$ and $0.05 \pm 0.05$ were determined in ascites for the liberated and the lipid- formulated $\mathrm{AmB}$ fraction, respectively. Liberated AmB could be detected in all samples of pleural effusion $(0.02$ to $0.40 \mathrm{~g} / \mathrm{L})$, ascites $(0.15$ to $0.36 \mathrm{~g} / \mathrm{L})$ and bile fluid $(0.78$ $\mathrm{g} / \mathrm{L})$. Total AmB levels in pleural effusion and bile fluid were significantly lower than the total concentrations in plasma samples $(0.40$ to $6.91 \mathrm{~g} / \mathrm{L})$. Concentrations of the lipid-associated fractions were very low in body fluids $(<0.11 \mathrm{~g} / \mathrm{L})$. AmB concentrations in pleural effusions correlated positively with the cumulative dose $(\mathrm{r}=0.96, \mathrm{p}=$ $0.01)$.

\section{Conclusion}

After treatment with lipid formulations, AmB concentrations in pleural effusion and in ascites were substantially lower in body fluids than in plasma samples and human tissue. Long-term treatment with high doses of AmB lipid formulations as well as additional therapeutic interventions will be required for the eradication of fungal pathogens from body fluids. 